\title{
Solid-liquid transition of ultrathin lubricant film
}

\author{
Alexei V. Khomenko* and Olga V. Yushchenko \\ Physical Electronics Department, Sumy State University, 40007 Sumy, Ukraine
}

(Received 24 March 2003; published 12 September 2003)

\begin{abstract}
We represent a melting of ultrathin lubricant film by friction between atomically flat surfaces as a result of action of spontaneously appearing elastic field of stress shear component caused by the external supercritical heating. The kinetics of this solid-liquid transition is described by the Maxwell-type and Voigt-Kelvin equations for viscoelastic matter as well as by the relaxation equation for temperature. We show that these equations coincide formally with the synergetic Lorenz system, where the stress acts as the order parameter, the conjugate field is reduced to the elastic shear strain, and the temperature is the control parameter. Using the adiabatic approximation we find the steady-state values of these quantities. Taking into account the deformational defect of the shear modulus, we show that lubricant melting is realized according to mechanism of the first-order transition. The critical temperature of the friction surfaces increases with growth of the characteristic value of shear viscosity and decreases with growth of the shear modulus value linearly.
\end{abstract}

DOI: 10.1103/PhysRevE.68.036110

PACS number(s): 64.60.-i, 62.20.Fe, 62.20.Qp, 68.60.-p

\section{INTRODUCTION}

The interest in the problem of sliding friction is due to its applied engineering importance [1]. One of the main goals of studies in this field is to define the conditions for low friction. In this direction experiments were carried out with atomically flat mica surfaces separated by ultrathin layer of liquid lubricant that manifested a solid-type behavior at defined experimental conditions [2]. In particular, the stick-slip (or interrupted) motion has been observed at critical yield stress inherent in solid friction. This effect occurs when the lubricant film consists of several molecular layers and is explained as a confinement-induced freezing. The resulting melting takes place when shear stress is above some critical value due to "shear-induced melting" effect. Numerical method studies $[3,4]$ maintain that liquid molecular ordering is due to wall confinement. Studies described in [5-7] were initiated for quantitative description of experimental results. In particular, in Ref. [7] the lubricant behavior was described using the order parameter determining the melting degree. On the basis of viscoelastic matter approximation and Ginzburg-Landau equation, where order parameter defines the shear melting and freezing, the observed phenomenology of ultrathin fluid film in the process of friction is successfully described in Ref. [8]. Here the phase diagram is calculated defining the domains of sliding, stick-slip, and dry friction in the plane temperature-film-thickness.

The starting point of our approach is the synergetic concept of phase transition $[9,10]$, which is the generalization of phenomenological Landau theory. According to the latter, the phase transition is controlled by an order parameter, over which value $\phi$ a free energy $F$ is developed in a power expansion [11]. The equilibrium value of $\phi$ is determined by the condition

$$
\partial F / \partial \phi=h,
$$

*Electronic address: khom@phe.sumdu.edu.ua where $h$ is the external field and $F$ is the free energy at $h$ $=0$. The relaxation process of transition to equilibrium is described by the equation [12]

$$
\dot{\phi}=-\frac{1}{\eta}\left(\frac{\partial F}{\partial \phi}-h\right) .
$$

Here $\eta$ is the kinetic coefficient, which can be considered as the generalized viscosity. If $\phi$ is close to its equilibrium value $\phi_{0}=0$, we can use the linear approximation $\partial F / \partial \phi$ $\approx \phi / \chi$, where $\chi \equiv \partial \phi / \partial h=\left(\partial^{2} F / \partial \phi^{2}\right)^{-1}$ is the susceptibility. As a result, the relaxation equation (2) takes the linear form

$$
\tau \dot{\phi}=-\phi+\chi h,
$$

where

$$
\tau=\chi \eta
$$

is the relaxation time.

Equations (3) and (4) were used by Landau and Khalatnikov to study the anomalous ultrasound absorption in the vicinity of phase transition. They held the fact that here susceptibility $\chi \rightarrow \infty$ and supposed that viscosity $\eta$ is practically independent of temperature $T$. In their theory the anomalously large magnitude of $\tau$ is responsible for freezing process.

For viscoelastic matter the shear modulus $G$ plays a role of the inverse susceptibility and expression (4) assumes the form

$$
\tau=\eta / G .
$$

In the cases of viscoelastic and displacement-type phase transitions (for example, of martensite type) the modulus $G$ goes to zero in the vicinity of transition point and relaxation time (5) diverges $[13,14]$. There are studies (see, for example, Ref. [15]), using the fact that generalized susceptibility is practically independent of temperature but viscosity strongly increases with temperature at glass transition. Let us note 
that anomalously large value of equilibrium coefficient (susceptibility) is typical for phase transition and anomalously large value of kinetic coefficient is inherent in glass transition.

We are aiming to take into account, along the line [8], that the solid-liquid transition of ultrathin lubricant film occurs due to both thermodynamic and shear melting. We obtain the unified analytical description of these processes as a result of the self-organization caused by the positive feedback of shear stress and temperature on shear strain on the one hand, as well as the negative feedback of shear stress and strain on temperature on the other hand. Our approach is based on the assumption that relaxation time $\tau \rightarrow \infty$ because the shear viscosity diverges at the point of transition. The feature of using the synergetic approach is that it allows us to obtain the synergetic potential, which is the analog of free energy, from some simple equations.

\section{BASIC EQUATIONS}

The main assumption of our approach is that relaxation equation of the shear component $\sigma$ of elastic stress tensor has the form similar to the Landau-Khalatnikov equation (3):

$$
\tau_{\sigma} \dot{\sigma}=-\sigma+G \varepsilon
$$

Here $\varepsilon$ is the corresponding component of strain, the first term on the right-hand side describes the Debye relaxation during time $\tau_{\sigma} \equiv \eta_{\sigma} / G$ determined by values of effective viscosity $\eta_{\sigma}$ and shear modulus $G$. In the stationary case $\dot{\sigma}$ $=0$ the kinetic equation (6) is transformed into the Hooke law

$$
\sigma=G \varepsilon
$$

Relaxation behavior of viscoelastic matter is described also by the Voigt-Kelvin equation [16]

$$
\dot{\varepsilon}=-\varepsilon / \tau_{\varepsilon}+\sigma / \eta,
$$

where $\tau_{\varepsilon}$ is the relaxation time of matter strain and $\eta$ is the shear viscosity coefficient. The second term on the righthand side describes the flow of a viscous liquid caused by the shear components of the elastic stress. In the stationary case $\dot{\varepsilon}=0$ we obtain the Hooke-type expression $\sigma=G_{\varepsilon} \varepsilon$. It is worth noting that effective values of viscosity $\eta_{\sigma} \equiv \tau_{\sigma} G$ and modulus $G_{\varepsilon} \equiv \eta / \tau_{\varepsilon}$ do not coincide with the real values $\eta$ and $G$. The formal reason for this difference is that the Maxwell-type equation (6) does not reduce to the VoigtKelvin equation (8) [17]. It is very important for our consideration that the values $G_{\varepsilon}, G, \eta_{\sigma}$ depend on temperature $T$ very weakly, while the real viscosity $\eta$ diverges, if the temperature decreases to point $T_{c}$ [15]. Further, we will use the simplest approximate temperature dependencies: $G_{\varepsilon}(T), G(T), \eta_{\sigma}(T)=$ const,

$$
\eta=\frac{\eta_{0}}{T / T_{c}-1}
$$

where $\eta_{0} \equiv \eta\left(T=2 T_{c}\right)$ is the typical value of viscosity.
According to synergetic ideology $[9,10]$ for completing the equation system (6) and (8), which contain the order parameter $\sigma$, the conjugate field $\varepsilon$, and the control parameter $T$, it is necessary to add a kinetic equation for the temperature. This equation can be obtained using the basic equations of elasticity theory stated in Ref. [17]. Thus, we should start from the equation connecting the time derivatives of entropy $S$ and internal energy $U$ with equilibrium stress $\sigma_{0}$,

$$
T \frac{d S}{d t}=\frac{d U}{d t}-\sigma_{0} \frac{d \varepsilon}{d t}
$$

(in equilibrium, the heat variation is $d Q=T d S$ ). In nonequilibrium case of nonuniform medium heating this equation has the form

$$
-\operatorname{div} \mathbf{q}=\frac{d U}{d t}-\sigma \frac{d \varepsilon}{d t}
$$

Here the heat current is given by the Onsager equation

$$
\mathbf{q}=-\kappa \boldsymbol{\nabla} T
$$

where $\kappa$ is the heat conductivity constant, and the total stress $\sigma=\sigma_{0}+\sigma^{\prime}$ includes the viscous part $\sigma^{\prime}$. Deducting Eq. (11) from Eq. (10), taking into account the equality

$$
\begin{aligned}
\frac{d S}{d t} & =\frac{\partial S}{\partial U}\left(\frac{\partial U}{\partial T}\right)_{\varepsilon} \frac{d T}{d t}+\frac{\partial S}{\partial U} \frac{\partial U}{\partial \varepsilon} \frac{d \varepsilon}{d t}+\left(\frac{\partial S}{\partial \varepsilon}\right) \frac{d \varepsilon}{d t} \\
& =\frac{\rho c_{v}}{T} \frac{d T}{d t}+\frac{1}{T} \frac{\partial U}{\partial \varepsilon} \frac{d \varepsilon}{d t}-\frac{\sigma_{0}}{T} \frac{d \varepsilon}{d t},
\end{aligned}
$$

and supposing that a layer of lubricant and atomically flat mica surfaces have different temperatures $T$ and $T_{e}$, respectively, we obtain

$$
\rho c_{v} \dot{T}=\frac{\kappa}{l}\left(T_{e}-T\right)+\sigma^{\prime} \dot{\varepsilon}+T \frac{\partial \sigma_{0}}{\partial T} \dot{\varepsilon} .
$$

Here the equalities $(\kappa / l)\left(T_{e}-T\right) \approx-\operatorname{div} \mathbf{q}$ and $\partial U / \partial \varepsilon=\sigma_{0}$ $-T \partial \sigma_{0} / \partial T$ are used, $l$ is the scale of heat conductivity, $\rho$ is the mass density, and $c_{v}$ is the specific heat capacity. The first term on the right-hand side of Eq. (14) describes the heat transfer from the layer of lubricant to friction surfaces. The second term takes into account the effect of the dissipative heating of a viscous liquid flowing under the action of the stress [18]. The third term represents the heat source that is conditioned by the reversible mechanic-and-caloric effect for which in linear approximation $T\left(\partial \sigma_{0} / \partial T\right) \dot{\varepsilon} \approx \sigma_{0} \dot{\varepsilon}$. As a result, the equation of heat conductivity can be written in the form

$$
\rho c_{v} \dot{T}=\frac{\kappa}{l}\left(T_{e}-T\right)+\sigma \dot{\varepsilon} .
$$

It is convenient to introduce the following measure units:

$$
\sigma_{s}=\left(\rho c_{v} \eta_{0} T_{c} / \tau_{T}\right)^{1 / 2}, \quad \varepsilon_{s}=1, \quad T_{c}
$$


for variables $\sigma, \varepsilon, T$, respectively $\left(\tau_{T} \equiv \rho l c_{v} / \kappa\right.$ is the time of heat conductivity). Then, substituting in Eq. (15) the expression for the $\dot{\varepsilon}$ from Eq. (8), the basic equations (6), (8), and (15) take the form

$$
\begin{gathered}
\tau_{\sigma} \dot{\sigma}=-\sigma+\left(g / \theta_{0}\right) \varepsilon, \\
\tau_{\varepsilon} \dot{\varepsilon}=-\varepsilon+\theta_{0}(T-1) \sigma, \\
\tau_{T} \dot{T}=\left(T_{e}-T\right)-\sigma \varepsilon / \theta_{0}+\sigma^{2},
\end{gathered}
$$

where we introduce the constants

$$
\begin{gathered}
g=\frac{G}{G_{0}}, \quad G_{0} \equiv \frac{\eta_{0}}{\tau_{\varepsilon}} ; \\
\theta_{0}=\frac{\sigma_{s}}{G_{0}} \equiv\left(\frac{\tau_{\varepsilon}}{\tau_{T}}\right)^{1 / 2}\left(\frac{\rho c_{v} T_{c} \tau_{\varepsilon}}{\eta_{0}}\right)^{1 / 2} .
\end{gathered}
$$

It is easy to see that Eqs. (17)-(19) have the form similar to the Lorenz system $[9,10]$.

\section{CONDITIONS OF TRANSITION}

For qualitative analysis of this system let us use the adiabatic approximation, when the characteristic time scales are submitted to the following inequalities:

$$
\tau_{\varepsilon} \ll \tau_{\sigma}, \quad \tau_{T} \ll \tau_{\sigma} .
$$

They mean that in the course of medium evolution the strain $\varepsilon(t)$ and the temperature $T(t)$ follow the change of the stress $\sigma(t)$. The first of these conditions compares the macroscopic time $\tau_{\sigma}$ and microscopic Debye time $\tau_{\varepsilon} \sim 10^{-12} \mathrm{~s}$, so it is satisfied always. Using the definitions of the thermometric conductivity $\chi \equiv \kappa / c_{v}$, the effective kinematic viscosity $\nu_{\sigma} \equiv \eta_{\sigma} / \rho$, and the sound velocity $c \equiv(G / \rho)^{1 / 2}$, it is convenient to give to the second condition (21) the form

$$
l \ll L,
$$

according to which the characteristic length of heat conductivity should not to exceed the value

$$
L=\frac{\chi \nu_{\sigma}}{\rho c^{2}} .
$$

Then, we can set the left-hand sides of Eqs. (18) and (19) to be equal to zero. As a result, the dependencies of the strain $\varepsilon$ and the temperature $T$ on the stress $\sigma$ read

$$
\begin{gathered}
\theta_{0}^{-1} \varepsilon=\sigma-\left(2-T_{e}\right) \frac{\sigma}{1+\sigma^{2}}, \\
T=T_{e}+\left(2-T_{e}\right) \frac{\sigma^{2}}{1+\sigma^{2}} .
\end{gathered}
$$

According to Eq. (24), at $T_{e}<1$ the $\varepsilon$ vs $\sigma$ dependence acquires a minimum at $\sigma=\sigma_{+}$, defined by the equality

$$
\sigma_{\mp}=\frac{1}{2}\left[\left(T_{e}-4\right) \mp \sqrt{\left(T_{e}-2\right)\left(T_{e}-10\right)}\right]^{1 / 2},
$$

and then increases monotonically [19]. When $T_{e}>1$ the strain $\varepsilon$ increases with stress $\sigma$ linearly at Hook domain $\sigma$ $\ll 1$. For values of $T_{e}$ in the $(1,10)$ interval, the $\varepsilon$ vs $\sigma$ dependence has a monotonically increasing shape with its minimum at point $\sigma=0$. At $T_{e}=10$ a plateau appears, which for $T_{e}>10$ is transformed into a maximum and a minimum corresponding to the stresses $\sigma_{-}$and $\sigma_{+}$, respectively. The temperature $T$ at $T_{e}<2$ increases with stress $\sigma$ from the temperature $T_{e}$ at $\sigma=0$ to the horizontal asymptote $T=2$, and at $T_{e}>2$ control parameter decreases from the maximum value $T_{e}$ at $\sigma=0$ to the same asymptote $T=2$. Obviously, this decrease is caused by the negative feedback of the stress $\sigma$ and the strain $\varepsilon$ on the temperature $T$ in Eq. (19), which is the reflection of Le Chatelier principle for the examined problem. On the other hand, the positive feedback of the stress $\sigma$ and the temperature $T$ on the strain $\varepsilon$ in Eq. (18) is the reason for melting that leads to the growth of $\varepsilon$ due to solid phase instability. However, in accordance with Eq. (19), the latter results in decrease of $T$ as a consequence of self-organization process.

The pointed out positive feedback of $\sigma$ and $T$ on $\varepsilon$ implies that the transition of lubricant from solid to fluid state is induced both by heating and under influence of stress generated by solid surfaces at friction. This agrees with examination of solid state instability within the framework of shear and dynamic disorder-driven melting representation in absence of thermal fluctuations [8].

The insertion of Eq. (24) into Eq. (17) gives the LandauKhalatnikov-type equation

$$
\tau_{\sigma} \dot{\sigma}=-\partial V / \partial \sigma,
$$

where the synergetic potential reads

$$
V=\frac{1}{2}(1-g) \sigma^{2}+g\left(1-\frac{T_{e}}{2}\right) \ln \left(1+\sigma^{2}\right) .
$$

At steady state the condition $\dot{\sigma}=0$ is realized and potential (28) assumes a minimum. If the temperature $T_{e}$ is smaller than the critical value

$$
T_{c}=1+g^{-1} ; \quad g \equiv G / G_{0}<1, \quad G_{0} \equiv \eta_{0} / \tau_{\varepsilon},
$$

this minimum corresponds to the stress $\sigma=0$, so that the melting cannot take place and the solid state is realized. In the opposite case $T_{e}>T_{c}$, the stationary shear stress has the nonzero value

$$
\sigma_{0}=\left(\frac{g T_{e}-(g+1)}{1-g}\right)^{1 / 2},
$$

increasing with $T_{e}$ growth according to the root law. This causes the melting of film and its transition into fluid state. In accordance with Eqs. (24) and (25), the corresponding stationary values of melting strain and temperature are as follows: 


$$
\varepsilon_{0}=\left(\sigma_{s} / G\right) \sigma_{0}, \quad T_{0}=1+g^{-1} .
$$

It is interesting that, on the one hand, the melting temperature $T_{0}$ coincides with critical value (29) and, on the other hand, its value differs from the temperature $T_{e}$. The latter circumstance takes place due to the fact at steady state the first equation (31) is realized instead of connection $\sigma_{0}$ $=\varepsilon_{0} / \theta_{0}$. Since $T_{c}$ is the minimum value of temperature at which a solid-liquid transition begins, the above means that the negative feedback of the elastic stress $\sigma$ and the strain $\varepsilon$ on the temperature $T$ [see third term on the right-hand side of Eq. (19)] reduces the film's temperature so much that only in the limit does it ensure the self-organization process. At steady state the melting value of shear viscosity coefficient is

$$
\eta_{m}=\eta_{0} g
$$

There are two opposite situations depending on the parameter $g=G / G_{0}$ value. In the case $g \gg 1$, this is realized for small value of the viscosity coefficient $\eta_{0}$, and Eqs. (29)(31) take the form

$$
\sigma_{0}=\left(1-T_{e}\right)^{1 / 2}, \quad T_{0}=T_{c}=1 .
$$

Such situation corresponds to the limit of strongly viscous liquid. In the opposite case $g \ll 1$ (large viscosity coefficient $\eta_{0}$ ) we have, instead of Eq. (33), the solid (fragile) limit,

$$
\sigma_{0}=\left(g T_{e}-1\right)^{1 / 2}, \quad T_{0}=T_{c}=g^{-1} \equiv \eta_{0} / \tau_{\varepsilon} G
$$

\section{INFLUENCE OF DEFORMATIONAL DEFECT OF MODULUS}

The Maxwell equation (6) assumes the use of the idealized Genki model. For the dependence $\sigma(\varepsilon)$ of the stress on the strain, this model is represented by the Hooke expression $\sigma=G \varepsilon$ at $\varepsilon<\varepsilon_{m}$ and the constant $\sigma_{m}=G \varepsilon_{m}$ at $\varepsilon \geqslant \varepsilon_{m}\left[\sigma_{m}\right.$, $\varepsilon_{m}$ are the maximal stress and strain, $\sigma>\sigma_{m}$ leads to viscous flow with the deformation rate $\left.\dot{\varepsilon}=\left(\sigma-\sigma_{m}\right) / \eta\right]$. Actually, the dependence $\sigma(\varepsilon)$ curve has two regions: first one, Hookean, has the large slope fixed by the shear modulus $G$, and it is followed by the more gently sloping section of the plastic deformation whose tilt is defined by the hardening factor $\Theta$ $<G$. Obviously, the above picture means that the shear modulus, introduced (in terms of the relaxation time $\tau_{\sigma}$ ) in Eq. (6), depends on the stress value. We use the simplest approximation

$$
G(\sigma)=\Theta+\frac{G-\Theta}{1+\sigma / \sigma_{p}},
$$

which describes the above represented transition of the elastic deformation mode to the plastic one. It takes place at characteristic value of the stress $\sigma_{p}$, which does not exceed the value $\sigma_{s}$ (in other case the plastic mode is not manifested). As a result, the relaxation time $\tau_{\sigma}$ obtains the dependence on the stress value:

$$
\frac{1}{\tau_{\sigma}(\sigma)}=\frac{1}{\tau_{p}}\left(1+\frac{\theta^{-1}-1}{1+\sigma / \sigma_{p}}\right),
$$

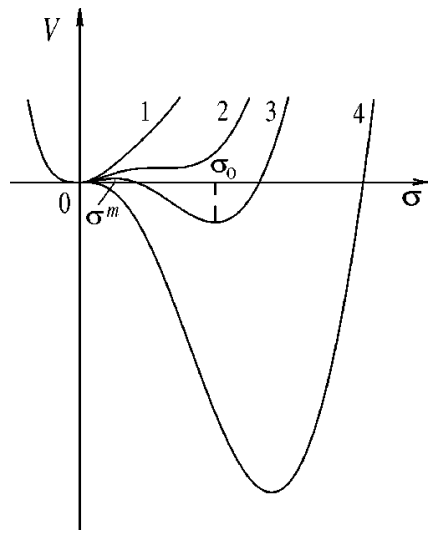

FIG. 1. The dependence of the synergetic potential on the elastic stress at various temperatures: (curve 1) $T_{e}<T_{c}^{0}$, (curve 2) $T_{e}$ $=T_{c}^{0}$, (curve 3) $T_{c}^{0}<T_{e}<T_{c}$, and (curve 4) $T_{e} \geqslant T_{c}$.

where the relaxation time is introduced for the plastic flow mode [cf. Eq. (5)],

$$
\tau_{p}=\eta_{\sigma} / \Theta
$$

and the quantity

$$
\theta=\Theta / G<1
$$

is the parameter describing the ratio of the tilts for the deformation curve on the plastic and the Hookean domains. Note that the expression of the type Eq. (36) was offered, for the first time, by Haken [9] for the description of the rigid mode of the laser radiation. We used it [10] for the description of the first-order phase transition kinetics, however, Eq. (36) had contained the square of the ratio $\sigma / \sigma_{p}$ (so the $V$ vs $\sigma$ dependence had the even form in Ref. [10]). In description of the structural phase transitions of a liquid the third-order invariants, breaking the specified parity, is present [11]. Therefore in approximation (36) we used the linear term $\sigma / \sigma_{p}$, instead of the square one $\left(\sigma / \sigma_{p}\right)^{2}$. It is apparent that in the following, dependence (39) is not already even.

Within the adiabatic approximation (21) the system of the Lorenz equations (17)-(19), where instead of the $\tau_{\sigma}$ it is necessary to use dependence $\tau_{\sigma}(\sigma)$, is reduced to, as well as above of, the Landau-Khalatnikov equation (27) with $\tau_{p}$ instead of $\tau_{\sigma}$. However, in the synergetic potential (28) the factor $g=G / G_{0}$ is replaced by $g_{\Theta}=G^{2} / G_{0} \Theta<1$, which is formally supposed to be not dependent on $\sigma$, and the odd term appears proportional to $\theta^{-1}-1$ :

$$
\begin{aligned}
V= & \frac{1}{2}\left(1-g_{\Theta}\right) \sigma^{2}+g_{\Theta}\left(1-\frac{T_{e}}{2}\right) \ln \left(1+\sigma^{2}\right)+\alpha^{2}\left(\theta^{-1}-1\right) \\
& \times\left(\frac{\sigma}{\alpha}-\ln \left|1+\frac{\sigma}{\alpha}\right|\right)
\end{aligned}
$$

Here the constant $\alpha \equiv \sigma_{p} / \sigma_{s}$ is introduced. At small value of temperature $T_{e}$ dependence Eq. (39) has a monotonically increasing shape with its minimum at point $\sigma=0$ corresponding to steady state of a solid. As it is seen from Fig. 1 at values 


$$
\begin{aligned}
& T_{c}^{0}=1+\left(g_{\Theta}^{-1}-1\right)(2 \sqrt{p / 3} \operatorname{ctg}(2 \delta)+r / 3) ; \\
& \operatorname{tg} \delta=\operatorname{tg}^{1 / 3}(\beta / 2)(|\delta| \leqslant \pi / 4), \quad \operatorname{tg} \beta=\frac{2}{q} \sqrt{\left(\frac{p}{3}\right)^{3}} \quad(|\beta| \leqslant \pi / 2), \\
& p=s-\frac{r^{2}}{3}, \quad q=\frac{2 r^{3}}{3^{3}}-\frac{r s}{3}+t, \\
& r=3^{3}\left(\frac{1}{3^{2}\left(1-g_{\Theta}\right)}+\frac{\alpha^{2}}{2^{2}}-\frac{\gamma^{2}}{2^{2} \times 3^{3}}-\frac{\gamma \alpha}{2 \times 3}\right), \quad \gamma=\frac{\alpha\left(\theta^{-1}-g_{\Theta}\right)}{1-g_{\Theta}}, \\
& s=3^{3}\left[\frac{1}{\left(1-g_{\Theta}\right)}\left(\frac{1}{3^{2}\left(1-g_{\Theta}\right)}+\frac{\alpha^{2} \theta^{-1}}{2}-\frac{\gamma^{2}}{2 \times 3^{3}}-\frac{\gamma \alpha \theta^{-1}}{2 \times 3}-\frac{\gamma \alpha}{2 \times 3}\right)+\frac{\gamma^{3} \alpha}{3^{3}}\right], \\
& t=3^{3}\left[\frac{1}{\left(1-g_{\Theta}\right)^{2}}\left(\frac{1}{3^{3}\left(1-g_{\Theta}\right)}+\frac{\alpha^{2} \theta^{-2}}{2^{2}}-\frac{\gamma^{2}}{2^{2} 3^{3}}-\frac{\gamma \alpha \theta^{-1}}{2 \times 3}\right)+\frac{\gamma^{3} \alpha \theta^{-1}}{3^{3}\left(1-g_{\Theta}\right)}\right],
\end{aligned}
$$

a plateau appears, which for $T_{e}>T_{c}^{0}$ is transformed into a minimum meeting the stress $\sigma_{0} \neq 0$ and a maximum $\sigma^{m}$ that separates minima corresponding to the values $\sigma=0$ and $\sigma$ $=\sigma_{0}$. With further growth of the temperature $T_{e}$ the "ordered" phase minimum, corresponding to a fluid state $\sigma$ $=\sigma_{0}$, grows deeper, and the height of the interphase barrier decreases, vanishing at the critical value $T_{c}=1+g^{-1}$ (29). The steady-state values of the stress in a fluid state have the form (see Figs. 1 and 2)

$$
\begin{gathered}
\sigma_{0}=2 \epsilon \cos \left(\frac{\varphi}{3}\right)-\frac{\gamma}{3}, \\
\sigma^{m}=2 \epsilon \cos \left(\frac{\varphi}{3}-\frac{2 \pi}{3}\right)-\frac{\gamma}{3} ; \\
\epsilon \equiv(-\psi / 3)^{1 / 2}, \quad \cos \varphi \equiv-\omega / 2 \epsilon^{3}, \\
\psi=\zeta-\frac{\gamma^{2}}{3}, \quad \omega=\frac{2 \gamma^{3}}{3^{3}}-\frac{\gamma \zeta}{3}+\xi,
\end{gathered}
$$

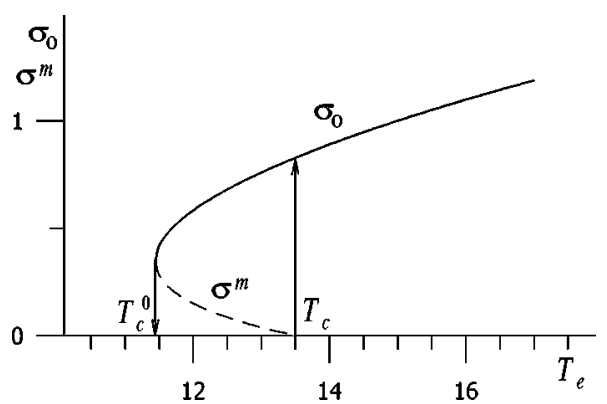

FIG. 2. The dependence of the steady-state values of the stress on the temperature $T_{e}$ at $g_{\Theta}=0.2, \theta=0.4$, and $\alpha=0.5$ (the solid curve corresponds to the steady-state value $\sigma_{0}$, the dashed curve meets the unstable one, $\sigma^{m}$ ).

$$
\begin{gathered}
\gamma=\frac{\alpha\left(\theta^{-1}-g_{\Theta}\right)}{1-g_{\Theta}}, \quad \zeta=\frac{1+g_{\Theta}\left(1-T_{e}\right)}{1-g_{\Theta}}, \\
\xi=\frac{\alpha\left[\theta^{-1}+g_{\Theta}\left(1-T_{e}\right)\right]}{1-g_{\Theta}} .
\end{gathered}
$$

At $T_{e} \geqslant T_{c}$ the dependence $V(\sigma)$ has the same character as in the absence of the modulus defect (see curve 4 in Fig. 1).

The specified peculiarities corresponds to the positive stress values $\sigma$. On the negative half-axis at $T_{e}>T_{c}$, with the increase of $|\sigma|$ a very weak minimum of the $V$ vs $\sigma$ dependence is observed, which is followed by the infinite increase of the potential $V$ at $\sigma=-\sigma_{p}$. Thus, for the negative values of the elastic fields $\sigma, \varepsilon$ are not realized practically.

The characteristic circumstance of our scheme is that energy barrier inherent in the synergetic first-order transition is displayed only in the presence of the deformational defect of the modulus. Since latter takes place always, it follows that a studied solid-liquid transition represents synergetic firstorder transition. The examined situation is much more complex than usual phase transitions. Really, in the latter case the steady-state value of the system's temperature $T_{0}$ is reduced to the value $T_{e}$ fixed by thermostat. In our case $T_{0}$ is reduced to the critical value $T_{c}$ for the synergetic second-order transition that has a place in the absence of the modulus defect (see Sec. III). When we take into account the modulus defect, the temperature

$$
T_{0}=T_{e}+\left(2-T_{e}\right) \frac{\sigma_{0}^{2}}{1+\sigma_{0}^{2}}
$$

is realized, whose value is defined by a minimum position of the dependence (39). According to Eqs. (41) and (43), the quantity $T_{0}$ smoothly decreases from the value 


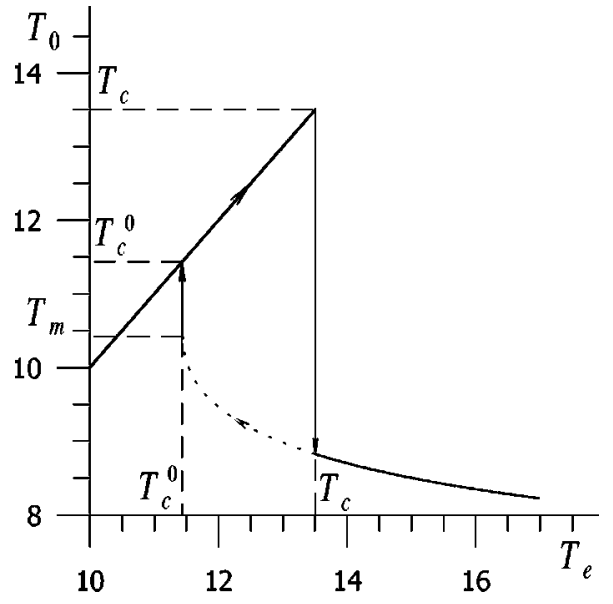

FIG. 3. The dependence of the steady-state value of the system temperature $T_{0}$ on the temperature $T_{e}\left(g_{\Theta}=0.2, \theta=0.4\right.$, and $\alpha$ $=0.5$ ).

$$
\begin{gathered}
T_{m}=T_{c}^{0}+\left(2-T_{c}^{0}\right) \frac{\left(\sigma_{0}^{c}\right)^{2}}{1+\left(\sigma_{0}^{c}\right)^{2}} \\
\sigma_{0}^{c}=\left[\frac{1}{3^{1 / 2}}\left(\frac{\gamma^{2}}{3}-\frac{1+g_{\Theta}\left(1-T_{c}^{0}\right)}{1-g_{\Theta}}\right)^{1 / 2}-\frac{\gamma}{3}\right]^{2}
\end{gathered}
$$

at $T_{e}=T_{c}^{0}$, to $T_{c 0} \equiv 1+g_{\Theta}^{-1}$ at $T_{e} \rightarrow \infty$. Referring to Fig. 3, the stationary temperature $T_{0}$ shows a linear increase from 0 to $T_{c}$, with $T_{e}$ being in the same interval and, after the jump down at $T_{e}=T_{c}$, the magnitude $T_{0}$ smoothly decays. If the temperature $T_{e}$ then decreases, the stationary temperature $T_{0}$ grows. When the point $T_{c}^{0}$ [Eq. (40)] is reached, $T_{0}$ undergoes the jump from $T_{m}$ [Eq. (44)] up to $T_{c}^{0}$. For $T_{e}<T_{c}^{0}$, again stationary temperature $T_{0}$ does not differ from $T_{e}$.
Since the stationary values of stress $\sigma_{0}>0$ are realized only at $T_{e}>1+g_{\Theta}^{-1}, g_{\Theta}<1$, the range of values $T_{c}^{0}>2$ is important for consideration. In this interval the maximum system's temperature (44) is lower than the minimum temperature of friction surfaces (40), and as it is visible from Fig. 3, at $T_{e}>T_{c}^{0}$ the stationary temperature $T_{0}$ of the film is always lower than value $T_{e}$.

\section{SUMMARY}

The above analysis is based on the assumption that a lubricant melting process is caused by the self-organization of the shear components of the stress and the strain elastic fields, on the one hand, and the lubricant temperature, on the other hand. Thus, the stress $\sigma$ acts as the order parameter, the conjugate field is reduced to the elastic strain $\varepsilon$, and the temperature $T$ is the control parameter. The initial reason for self-organization is the positive feedback of $T$ and $\sigma$ on $\varepsilon$ [see Eq. (18)]. According to Eqs. (8) and (9), it is caused by the temperature dependence of the shear viscosity leading to its divergence. Accounting for the deformational defect of the shear modulus, we obtain the expressions for temperatures corresponding to absolute instability of overcooled liquid $T_{c}^{0}$ [Eq. (40)] and stability limit of the solid state $T_{c}$ [Eq. (29)]. The real thermodynamic melting temperature is in the $\left(T_{c}^{0}, T_{c}\right)$ interval and can be found from the equality condition of potentials of solid and liquid phases, $V(0)=V\left(\sigma_{0}\right)$. It is seen from Eq. (29) that systems predisposed to melting have large shear modulus $G$ and small characteristic value of shear viscosity $\eta_{0}$.

The kinetics of a considered transition is determined by the Landau-Khalatnikov equation (27), where $\tau_{\sigma}$ is replaced by $\tau_{p}=\eta_{\sigma} / \Theta$ and the synergetic potential has the form (39) inherent in the first-order transition. In supercooled liquid with $\eta_{\sigma}=\infty$ the freezing of system can takes place $(\dot{\sigma} \rightarrow 0)$ even in the nonstationary state $\partial V / \partial \sigma \neq 0$.
[1] B.N.J. Persson, Sliding Friction. Physical Principles and Applications (Springer-Verlag, Berlin, 1998).

[2] H. Yoshizava and J. Israelachvili, J. Phys. Chem. 97, 4128 (1993).

[3] P.A. Thompson and M.O. Robbins, Phys. Rev. A 41, 6830 (1990).

[4] J.P. Gao, W.D. Luedtke, and U. Landman, J. Chem. Phys. 106, 4309 (1997).

[5] O. Braun, A.R. Bishop, and J. Röder, Phys. Rev. Lett. 82, 3097 (1999).

[6] W. Zhong and D. Tomanek, Europhys. Lett. 15, 887 (1991).

[7] J.M. Carlson and A.A. Batista, Phys. Rev. E 53, 4153 (1996).

[8] I.S. Aranson, L.S. Tsimring, and V.M. Vinokur, Phys. Rev. B 65, 125402 (2002).

[9] H. Haken, Synergetics. An Introduction. Nonequilibrium Phase Transitions and Self-Organization in Physics, Chemistry, and Biology, 3rd ed. (Springer, Berlin, 1983).

[10] A.I. Olemskoi and A.V. Khomenko, Zh. Éxp. Teor. Fiz. 83,
2144 (1996) [JETP 83, 1180 (1996)].

[11] L.D. Landau and E.M. Lifshitz, Statistical Physics, 3rd ed. (Pergamon Press, Oxford, 1980), Pt. 1.

[12] E.M. Lifshits and L.P. Pitaevskii, Phizicheskaia Kinetika (Physical Kinetics) (Nauka, Moscow, 1979) (in Russian).

[13] A.A. Kaznel'son and A.I. Olemskoi, Microscopic Theory of Nonhomogeneous Structures (Mir, Moscow, 1990).

[14] V.G. Bar'yahtar, A.I. Olemskoi, Fiz. Tverd. Tela 33, 2705 (1991).

[15] A. Havranek and M. Marvan, Ferroelectrics 176, 25 (1996).

[16] Rheology, edited by F.R. Eirich (Academic Press, New York, 1960).

[17] L.D. Landau and E.M. Lifshitz, Teorija Uprugosti (Elasticity Theory) (Nauka, Moscow, 1987).

[18] A.I. Olemskoi and A.V. Khomenko, Zh. Tekh. Fiz. 70, 6 (2000); Zh. Tekh. Fiz. 70, 10 (2000).

[19] The decrease domain of $\varepsilon(\sigma)$ function has no physical meaning. 